\title{
Principle, Application and Development of Heat Pipe Technology
}

\author{
Yuxue Liu', a \\ ${ }^{1}$ School of Geoscience and Technology, Southwest Petroleum University \\ aemail:liusuanyatong@126.com
}

Keywords: Heat Pipe Technology, Numerical Simulation, Experimental Study, enhanced heat transfer.

\begin{abstract}
Heat pipe technology is a rising new technology in recent decades, widely used in various aspects of engineering aspects and industrial production. In this paper, through literature research, the principle of heat pipe technology, classification, development history and research situation at home and abroad were summarized, points out the development direction of the technology.
\end{abstract}

\section{Introduction}

With the rapid development of technology in modern society, people's demand for energy and the Japanese surge, followed by the energy crisis and environmental pollution. How to effectively alleviate the energy crisis and reduce environmental pollution and achieve sustainable development become an important task. Energy conservation has become an important means to solve the issue of this series. Heat transfer enhancement to maximize the efficient use of energy, reducing waste, and thus more and more attention in the current study.

In the industrial production process, involving a large number of chemical reactions and energy-consuming equipment, and these reactions and devices will generate a lot of waste heat from waste energy, waste of resources and pollute the environment. In our daily running various boiler, heat exchanger, combustion equipment thousands of these devices generate a lot of energy consumption, much of it not be directly used, and released into the environment, resulting in a waste of energy and pollution of the environment, exacerbated by the greenhouse effect. Heat pipe as a low thermal resistance and efficient heat transfer components, the use of phase change heat, heat capacity is thousands of times the metal, no external power, can more effectively solve the above problems, and is widely used in chemical, building materials, textile, food, metallurgy, power engineering, electrical and electronic engineering, nuclear engineering, geotechnical engineering, petroleum engineering, aerospace and other fields.I

\section{Principle of Heat Pipe Technology}

Heat pipe technology in recent decades the rise of new technology, widely used in various aspects of real life and industrial production, the demand for heat pipe is also increasing. In practice, the majority of the heat pipe is working at a temperature below $200^{\circ} \mathrm{C}$, water, various ketones, various alcohols, ammonia, sodium metal, freon its main working fluid, the tube itself is made essentially aluminum , copper, carbon steel, alloy steel, stainless steel [11, 12]. Compared to conventional heat transfer apparatus, since by means of phase change heat transfer, high heat transfer efficiency, is thousands of times ordinary metals, and has a simple structure, low manufacturing cost. Heat pipe thermal resistance is small, it can be implemented in a small temperature difference to achieve a large heat transfer, during the transmission of energy loss, the process can be run without external power, hence the "superconducting hot body" in the world. But now there is still the problem of using a shorter life expectancy.

\section{History of Heat Pipe Technology}

Overall development of the heat pipe can be divided into three stages: the first stage is 1836 - 1942 , this is the prototype stage heat pipe, making rough, vacuum processing is not carried out, poor heat 
transfer performance. The second stage of the 1960s to the 1970s, this stage is to provide a modern thermal management theory and concepts, this phase of the heat pipes are mainly used in aerospace, space technology to solve the problem. The third stage is 70 years later, this phase heat pipe technology to tell the development of universities, research institutes in each country, factories and enterprises have begun to actively explore and study of heat pipe technology, papers on heat pipe technology after another, frequent international exchanges, the heat pipe wider range of applications.

\section{Classification of Heat Pipe}

Due to the wide range of heat pipes, the different forms, the inner workings of different media, the structure is very different, so there are multiple classifications of heat pipe: deep cold pipe $\left(-273{ }^{\circ} \mathrm{C} \sim\right.$ $\left.0{ }^{\circ} \mathrm{C}\right)$, its working fluid is mainly ethane, methane, nitrogen, oxygen, neon, etc.), often warmed tube $(0$ ${ }^{\circ} \mathrm{C} \sim 250^{\circ} \mathrm{C}$, its working fluid is mainly ammonia, Freon, acetone, ethanol, methanol, and water), in the hot tube $\left(250^{\circ} \mathrm{C} \sim 450{ }^{\circ} \mathrm{C}\right.$, its working fluid Dowtherm, mainly naphthalene), high temperature heat pipe $\left(450{ }^{\circ} \mathrm{C} \sim 1000{ }^{\circ} \mathrm{C}\right.$, its working fluid is mainly mercury, Yan, potassium, sodium, Keng, etc.).

According to the power of liquid flowing back, the heat pipe can be divided into: rotating heat pipe (by means of centrifugal force working fluid reflux), electrohydrodynamic heat pipe (to achieve the working fluid to reflux under kinetic force polarization current body effect), electro-osmotic heat pipe (in realization effect electro-osmotic pressure of the working fluid reflux), gravity heat pipe (also known as two-phase closed thermosyphon achieve working fluid reflux under the action of gravity), gravity assisted heat pipe (on the basis of the wick on a certain inclination achieved in the auxiliary force of gravity working fluid reflux), there is a core of the heat pipe (use a wick to achieve the working fluid reflux), magneto-hydrodynamic heat pipe (to achieve the working fluid reflux under the action of an external magnetic field) and the like.

By combination shell and the working fluid is different, the heat pipe can be divided into (this is the division method a habit): copper-water heat pipe, steel-water heat pipe, copper-steel composite-water heat pipe, aluminum-acetone heat pipe, carbon Cai heat a steel pipe, stainless steel a sodium heat pipe.

Different form of the structure, the heat pipe can be divided into: ordinary heat pipe, separate type heat pipe, the capillary pump loop heat pipes, micro heat pipe, heat pipe flat, radial heat pipe.

According to different functions, the heat pipe can be divided into: heat pipe heat transfer, thermal diode, thermal switch, thermal control heat pipe, heat pipe simulation, refrigeration heat pipe.

\section{Features of Heat Pipe}

Heat pipe relies mainly on the heating cycle working fluid phase transition section to absorb a lot of heat, then condensation section of a gas refrigerant returns to liquid, releasing latent heat of vaporization to achieve heat transport. In simple terms, the heat pipe is a labor matter phase change method of heat from one end to the other end of the device. Usually the choice of working fluid having a heat pipe tube compatibility, good heat transfer performance, easy gasification characteristics.

Heat pipe features are as follows:

(1) Heat pipe can effectively improve the heat transfer coefficient, high thermal conductivity. High heat transfer coefficient, can reduce the volume occupied by the heat exchanger. Heat pipe can effectively improve the heat transfer coefficient for the following reasons: Heat pipe with the working fluid phase transition heat, latent heat of vaporization of the working medium large, small resistance, is thousands of times generally metallic heat transfer capabilities; Heat pipe can fins disposed evaporator and condenser section to the outer heat transfer enhancement; The working fluid flow of the heat pipe is relatively weak, the boundary layer is very thin, the thermal resistance is small. 
(2) Fast heat transfer: saturated steam pipe close to the local speed of sound, heat quickly.

(3) Diverse structure: according to the conditions of hot and cold sources need different forms of heat pipes is varied, heating and cooling section can also be arbitrarily chosen, which greatly broadens the field of application of heat pipe, heat pipe enhanced adaptability.

(4) Wide used temperature range: From the heat pipe according to temperature classification can be seen, the heat pipe in the range of $-273{ }^{\circ} \mathrm{C} \sim 1000{ }^{\circ} \mathrm{C}$ are applicable.

(4) Strong ability to transfer heat: Heat transfer heat pipe rely on the working fluid evaporation condensation phase change heat, this heat exchanger way better than other forms of heat exchange capacity, phase change heat transfer thousands of times stronger than the thermal conductivity of various metals.

(5) Good thermostat type: heat pipe inside the phase change process is a process of constant pressure and constant temperature. It Can deliver high heat in a small temperature difference: because can increase evaporation and condenser section fins, increasing the contact area, thereby increasing the heat transfer coefficient, which compared to other forms of heat at the same temperature, the heat pipe can transfer other forms of excess heat greatly.

(7) Low pressure loss: Heat pipe has been working at the operating temperature corresponding to the saturation pressure, can reduce fan power consumption.

(8) Replaceable components, structure simple: heat pipe element is an independent installation can be replaced individually as needed.

(9) No additional power: the flow by the heat pipe heat pipe is pressure due to heat transfer and is therefore working fluid cycle work without additional power when.

(10) Heat Pipe Technology can adjust the amount of heat transfer, with good adaptability. Because the heat pipe is by the latent heat of phase change to transmission of heat, latent heat is affected by pressure and temperature can be controlled by the pressure and temperature of the heat pipe to regulate the heat transfer.

\section{Research Status of Heat Pipe}

For current heat pipe research focused on four aspects: First, the method of theoretical analysis, many reasonable assumptions at the premise of the analytical solution within the heat pipe heat transfer flows. Second, the use of experimental methods, with the depth of the heat pipe research by experiment on the influence of heat pipe heat exchanger quantitative analysis of various factors, heat pipe heat transfer mechanism to clarify and strengthen the heat pipe heat exchanger; the third is the use of simulation technology approach to simulation of heat pipe. Because the heat pipe heat transfer mechanism complicated, because there are many restrictions experimental means, through numerical simulation approach can be better observed operating conditions inside the heat pipe can be obtained many experimental results cannot be obtained, and thus a better understanding of the mechanism of heat pipe heat exchanger . Fourth, the application of heat pipe, the results obtained in the first two aspects better used in various areas of practice, so as to maximize the energy use, save costs and improve production efficiency. In recent years, along with other new forms of heat pipe (nano-for-hot tube, magnetic fluid heat pipe, etc.) continue to emerge, the heat pipe coverage is gradually expanded.

\section{Conclusions}

Heat pipe technology will have an increasingly wide range of applications, mainly to study abroad means in future experiments based industrial, engineering and research and other fields. Numerical Simulation CFD software fluent mainly carried out, and need to customize function (UDF) to more accurately simulate gravity heat pipe phase transition process. On the basis of the traditional methods, new approaches such as artificial neural networks and other methods have been introduced to the study of which the heat pipe. 


\section{Acknowledgements}

This work was financially supported by the science and technology innovation seedling project in Sichuan Province (2015126).

\section{References}

[1] Grover G, Cotter T, Erickson G. Structures of very high thermal conductance[J]. Journal of Applied Physics, 1964,35(6): 1990-1991.

[2] Fadhl B, Wrobel LC, Jouhara H. CFD modelling of a two-phase closed thermosyphon charged with R134a and R404a[J]. Applied Thermal Engineering, 2015,78: 482-490

[3] Yu Le, Liu Jianjun. Stability of interbed for salt cavern gas storage in solution mining considering cusp displacement catastrophe theory. Petroleum, 2015, 1(1): 82-90

[4] Schmidt E. Heat transmission by natural convection at high centrifugal acceleration in water-cooled gas-turbine blades[J]. Proc General Discussion on Heat Transfer, 1951,4: 361-363

[5] Leyong Chen, Jianjun Liu. Numerical analysis on the crack propagation and failure characteristics of rocks with double fissures under the uniaxial compression. Petroleum, 2015,1(4):373-381

[6] Dai Xiaojun; Liu Jianjun; Li Yuan. Analysis of roadbed deformation monitoring of cangzhou section of Beijing to Shanghai express railway, Electronic Journal of Geotechnical Engineering, 20(2), pp 813-820

[7] Solomon AB, Roshan R, Vincent W, Karthikeyan V, Asirvatham LG. Heat transfer performance of an anodized two-phase closed thermosyphon with refrigerant as working fluid[J]. International Journal of Heat and Mass Transfer, 2015,82: 521-529

[8] Quanshu Li; Huilin Xing, Jianjun Liu, Xiangchon Liu. A Review on Hydraulic Fracturing of Unconventional Reservoir. Petroleum, 2015, 1(1):8-15

[9] Vafai K, Kim S-J. Analysis of surface enhancement by a porous substrate[J]. Journal of Heat Transfer, 1990,112(3): 700-706.

[10] Jianjun Liu, Qiang Xiao. The Influence of Operation Pressure on the Long-Term Stability of Salt-Cavern Gas Storage. Advances in Mechanical Engineering, 2014,9, Article ID:537679

[11] Gou Feifei, Liu Jianjun, Liu Weidong. Numerical Simulation of Chemical Transferring Using Finite Difference Method. Journal of Southwest Petroleum University(Natural Science Edition), 2015, 37(6):112-118

[12] Bu-Xuan W, Jian-Hua D. Forced convective heat transfer in a vertical annulus filled with porous media[J]. International Journal of Heat and Mass Transfer, 1993,36(17): 4207-4213

[13] Liu, Jianjun; Zhang, Huijuan. Experimental Study for Dynamic Mechanical Properties of Shale using Split Hopkinson Pressure Bar. Disaster Advances, 2013, 6(11):38-42

[14] Song Rui, Liu Jianjun. Study on Porosity and Permeability of Rock Core based on 3D CT Images and Structured Pore-Network Model. Journal of Southwest Petroleum University(Natural Science Edition), 2015, 37(3):138-145

[15] Wang Y-w, Lu T, Jiang P-x, Cheng P-f, Wang K-s. Large-eddy simulation of fluid mixing in tee with sintered porous medium[J]. Applied Mathematics and Mechanics, 2012,33: 911-922 
[16] Liu Jianjun, Song Rui, Zhao Jinzhou. Numerical Simulation Research on Seepage Mechanism in Pore-scale Deformable Porous Media. Disaster Advances, 2013, 6(S1): 49-58

[17] Heris S, Mohammadpur F, Mahian O, Sahin A. Experimental study of two phase closed thermosyphon using $\mathrm{CuO} /$ Water nanofluid in the presence of electric field[J]. Experimental Heat Transfer, 2015,28(4): 328-343.

[18] Liu Jianjun, Zhang Linzhi, Zhao Jinzhou. Numerical Simulation on Open Wellbore Shrinkage and Casing Equivalent Stress in Bedded Salt Rock Stratum. Scientific World Journal, 2013, Article ID:718196

[19] Liu Jianjun, Lin Lijun, Song Rui, Zhao Jinzhou. A Pore Scale Modelling of Fluid Flow in Porous medium Based on Navier-Stokes Equation. Disaster Advances, 2013, 6(S1): 129-136

[20] Kaviany M. Laminar flow through a porous channel bounded by isothermal parallel plates[J]. International Journal of Heat and Mass Transfer, 1985,28(4): 851-858.

[21] Liu Jianjun, Yu Xianbin, Xiong Huan, Zhang Linzhi. Influence of Perforation on the Wellbore Surrounding Rock Stress and Fracture Pressure. Science \& Technology Review, 2013, 31(4): 43-48

[22] Chikh S, Boumedien A, Bouhadef K, Lauriat G. Non-Darcian forced convection analysis in an annulus partially filled with a porous material[J]. Numerical Heat Transfer, Part A: Applications, 1995,28(6): 707-722.

[23] Liu Jian-Jun, Xie Jun. Numerical simulation of thermo-hydro-mechanical coupling around underground pipelines in patchy permafrost region. Rock and Soil Mechanics, 2013, 34(S1): 444-450

[24] Jian-Jun Liu, Xian-Bin Yu, Jin-Zhou Zhao. Numerical simulation of geostress and pore pressure evolution around oil or water well under different injection-production ratio. Mathematical Problems in Engineering, Vol.2013, Article ID:604748

[25] Dai Xiaojun, Liu Jianjun, Zhang Huaying. Application of AR Model in the Analysis of Preearthquake Ionospheric Anomalies. Mathematical Problems in Engineering, 2015, Article ID: 157184

[26] Jianjun Liu, Rui Song. Investigation of water and CO2 flooding using pore-scale reconstructed model based on micro-CT images of Berea sandstone core. Progress in Computational Fluid Dynamics, An International Journal. 2015,15(5):317-326

[27] Al-Nimr M, Abu-Hijleh B. Validation of thermal equilibrium assumption in transient forced convection flow in porous channel[J]. Transport in porous media, 2002,49(2): 127-138.

[28] Lu T, Jiang P, Guo Z, Zhang Y, Li H. Large-eddy simulations (LES) of temperature fluctuations in a mixing tee with/without a porous medium[J]. International Journal of Heat and Mass Transfer, 2010,53(21): 4458-4466.

[29] Wang Y, Wang P, Lu T. Experimental investigation of the thermal fluctuations in hot and cold fluids mixing in a T-junction filled with spherical particles[J]. Applied Thermal Engineering, 2014,71(1): 310-316.

[30] Liu Jianjun, Song Rui, Cui Mengmeng. Improvement of predictions of petrophysical transport behavior using three-dimensional finite volume element model with micro-CT images. Journal of Hydrodynamics. 2015,27(2):234-241

[31] Hajipour M, Dehkordi AM. Analysis of nanofluid heat transfer in parallel-plate vertical channels partially filled with porous medium[J]. International Journal of Thermal Sciences, 2012,55: 103-113. 
[32] Yang C, Nakayama A, Liu W. Heat transfer performance assessment for forced convection in a tube partially filled with a porous medium[J]. International Journal of Thermal Sciences, 2012,54: 98-108.

[33] Hamdan MO. An empirical correlation for isothermal parallel plate channel completely filled with porous media[J]. Thermal Science, 2013,17(4): 1061-1070.

[34] Pei Guihong, Yu Feng, Liu Jianjun. Feasibility Study of Individual Treatments of Desizing Wastewater. Journal of Residuals Science \& Technology. 2015, 12(S1):S85-S91

[35] Mahmoudi Y, Karimi N. Numerical investigation of heat transfer enhancement in a pipe partially filled with a porous material under local thermal non-equilibrium condition[J]. International Journal of Heat and Mass Transfer, 2014,68: 161-173.

[36] Ji Youjun, Liu Jianjun, Cheng Linsong. Feasibility Analysis of Steam Flooding with Horizontal Wells for Extra Heavy Oil Reservoir. Chinese Journal of Rock Mechanics and Engineering, 2013, 32(S2):3804-3811

[37] Liu Jianjun, Li Quanshu. Numerical Simulation of Injection Water Flow through Mudstone Interlayer in Low Permeability Oil Reservoir Development . Disaster Advances, 2012, 5(4): 1639-1645

[38] Ji You-Jun, Liu Jian-Jun, Cheng Lin-Song. Numerical simulation of tunnel excavation considering fluid solid coupling, Rock and Soil Mechanics, 2011, 32(4): 1229-1233

[39] Qin D. H., Liu L. L., Mao T., Liu J.J., Liu F.Z.. Corrosion behavior of 5Cr0.5Mo steel in sulfur-bearing solution at high temperature. Materialwissenschaft Und Werkstoftechnik, 2015, 46(9):978-982

[40] Liu Jianjun, Xie Jun. Numerical simulation of thermo-hydro-mechanical coupling around underground pipelines in patchy permafrost region. Rock \& Soil Mechanics, 2013, 34(S1): 444-450

[41] Torabi M, Zhang K, Yang G, Wang J, Wu P. Heat transfer and entropy generation analyses in a channel partially filled with porous media using local thermal non-equilibrium model[J]. Energy, 2015,82: 922-938. 